\title{
Validation of an automated cell counter to determine leukocyte differential counts in neonatal Holstein calves
}

\author{
T. E. von Konigslow, D. L. Renaud, T. F. Duffield, V. Higginson, and D. F. Kelton* \\ Department of Population Medicine, University of Guelph, Guelph, ON, Canada N1G 2W1
}

\section{ABSTRACT}

Recent advances in the understanding of risk factors and biomarkers in calves entering rearing facilities show promise for identifying high-risk calves on arrival at veal and dairy beef operations. Rapid automated leukocyte differential cell counts may be a good addition for augmenting or refining calf risk identification on-farm. The objective of this study was to validate an automated leukocyte cell counter, the QScout BLD test (Advanced Animal Diagnostics, Morrisville, NC), for its ability to determine leukocyte differential cell counts in neonatal Holstein calves. From June to July 2018, blood samples collected in EDTA anticoagulant from 235 calves upon arrival at an independent veal research facility in Ontario, Canada, were evaluated using the QScout BLD test and manually by microscopy. We compared these leukocyte differential counts using Lin's concordance correlation coefficient $(\rho)$ and found very good agreement between tests for neutrophil counts $(\rho=0.83)$; fair agreement for lymphocyte counts $(\rho=0.32)$; fair agreement for the ratio of neutrophils to lymphocytes $(\rho=0.36)$; slight agreement for monocyte counts $(\rho=$ 0.14 ); and slight agreement for eosinophil counts $(\rho=$ 0.20). We further examined test results to determine if they differed in their classification of samples as being above, within, or below reported $95 \%$ reference intervals for neonatal Holstein calves. Classification between tests resulted in very good agreement for neutrophils and lymphocytes, with only $4.2 \%$ and $5.8 \%$ disagreement in classification, respectively. We observed moderate agreement for monocytes, with $23.3 \%$ classified differently, and poor agreement for eosinophils, with $70.3 \%$ classified differently. Further study is required to determine the role of leukocyte profiling in the risk assessment of calves arriving at calf-rearing facilities.

Received January 25, 2019.

Accepted April 5, 2019.

*Corresponding author: dkelton@uoguelph.ca
Key words: calf, health indicator, leukocyte, differential

\section{INTRODUCTION}

Young calves entering veal and dairy beef facilities undergo several stressful events, including transportation, commingling, and variable periods of prolonged fasting. These stressors occur at an age when their immune and other physiological systems are still maturing (Marcato et al., 2018), which influences their susceptibility to disease. Calf mortality and morbidity in these facilities is known to be highest early in the production cycle (Bähler et al., 2012; Winder et al., 2016; Renaud et al., 2018a), causing large amounts of antimicrobials to be used for group metaphylaxis after arrival. This high level of antimicrobial use, particularly in the veal industry, has led to the development of antimicrobial resistance in pathogens, affecting the calves and their caretakers (Bos et al., 2013; Catry et al., 2016). In response to the development of antimicrobial resistance, there is increased focus on antimicrobial stewardship, including concerted efforts to reduce antimicrobial use where possible, without sacrificing animal or human health and welfare. With recent work focused on identifying clinically measurable risk factors that affect mortality in calves arriving at veal facilities (Winder et al., 2016; Marcato et al., 2018; Renaud et al., 2018a,b), it may be possible to reduce antimicrobial use after arrival by selectively treating only high-risk calves instead of the predominant practice of metaphylaxis at arrival when risk is unknown.

Several studies have identified biomarkers such as cholesterol, IgG, packed cell volume, and weight as indicators of future risk for mortality or morbidity in young calves when measured on arrival at veal facilities (Marcato et al., 2018; Renaud et al., 2018b). Bovine hematology could also be used as an aid to improve disease diagnosis and supplement clinical examinations (Mohri et al., 2007; Roland et al., 2014). Specifically, the ratio of neutrophils to lymphocytes $(\mathbf{N}: \mathbf{L})$ and overall leukocyte count may be good differentiators between stress, inflammation, and temporary fear or excitement 
(Jones and Allison, 2007; Tornquist and Rigas, 2011; Roland et al., 2014). If an abnormal leukogram proves to be an accurate indicator of risk in young calves, then a rapid, calf-side automated leukocyte differential test such as the QScout BLD (Advanced Animal Diagnostics, Morrisville, NC) could be a good addition to arrival screening protocols for identifying high-risk calves. Calves that arrive with identifiable risk factors for morbidity or mortality or an abnormal leukogram could then be identified and selectively treated with antimicrobials, supportive therapy, or both, leaving the other calves untreated and reducing overall antimicrobial use at this stage of the production cycle. The QScout BLD is currently the only on-farm automated leukocyte differential test with recent validation in Holstein dairy cows, reporting test agreement with manual white blood cell differential counts for neutrophils and lymphocytes, but no agreement for monocytes or eosinophils (Kull et al., 2018).

The objective of this study was to validate the QScout BLD for its ability to determine leukocyte differential counts in neonatal Holstein calves.

\section{MATERIALS AND METHODS}

\section{Study Design}

Calves were enrolled in this prospective cross-sectional diagnostic accuracy study at an independent grain-fed veal research facility in Ontario, Canada. The guideline for reporting diagnostic accuracy studies, STARD 2015, was followed (Korevaar et al., 2016). This study was conducted in accordance with the Animal Care Committee requirements of the University of Guelph (Animal Use Protocol \#3850).

\section{Animal Enrollment}

All calves arriving at the veal research facility between June 11 and July 26, 2018, were eligible to be enrolled. Calves were housed in 1 of 3 rooms of 80 individual calf pens each, with free access to water and grain. A total of 240 calves were enrolled, with health scoring and blood sampling performed on the day of arrival at the facility. Calves arrived at the facility early to mid-afternoon.

\section{Data and Sample Collection}

Calf health scoring was performed by 1 of 2 observers before sample collection. Observer 1 was a practicing veterinarian, and observer 2 was a research assistant trained by the veterinarian. The following health pa- rameters were scored: navel, cough, flank, hydration, and other health abnormalities. A description of the health parameters scored can be found in Table 1 . These health parameters represent a subset of those identified as risk factors associated with mortality in veal calves (adapted from Renaud et al., 2018a). The "other health abnormality" category was included to capture detectable health abnormalities that would otherwise not be recorded, such as an infected wound or heavy ocular discharge equivalent to score 3 on the respiratory scoring system developed by McGuirk and Peek (2014). Health score images and definitions were available on laminated sheets at the time of scoring, along with a metal dowel measuring $20 \mathrm{~mm}$ in diameter as a reference for assessing navel diameter. In addition to health parameters, rectal temperature was measured. An iPad (Apple Inc., Cupertino, CA) loaded with Qualtrics software (https://www.qualtrics.com/) was used to collect individual health scores and measurement data.

Blood samples were collected from each calf by jugular venipuncture into 2 vacuum tubes $(10-\mathrm{mL}$ EDTA tube and 10-mL serum tube) using an 18-gauge, 1-inch Vacutainer needle (BD Vacutainer, Fairfield, NJ) on a plastic hub. A separate 20- $\mu$ L EDTA blood collection device was used to collect blood by jugular venipuncture for use with the QScout BLD white blood cell differential machine. Blood samples were stored in a cooler with ice packs within minutes of collection. Blood samples in EDTA were submitted to the Animal Health Laboratory at the University of Guelph (Guelph, ON, Canada) for a manual white blood cell differential count performed by microscopy within 12 $\mathrm{h}$ of collection. Serum samples were centrifuged within $5 \mathrm{~h}$ of collection, and then stored in a refrigerator for serum total protein analysis within $12 \mathrm{~h}$ of collection.

\section{Test Methods}

The white blood cell differential QScout BLD test (software v1.0.6.20023, Advanced Animal Diagnostics) was run for each calf within minutes of blood collection at the farm. Samples were loaded from the proprietary QDraw blood collection device (version ASM9033C, Advanced Animal Diagnostics) into the well of a proprietary QScout BLD test slide cartridge (version ASM9041-C, Advanced Animal Diagnostics). The slide cartridge was mounted into the machine, and the automated microscopy was performed and analyzed through the proprietary software. Results were obtained within 1 min.

Manual white blood cell differential tests are considered the gold standard (Kim et al., 2014) and are therefore the most appropriate comparison for this 
Table 1. Description and prevalence [\% (n)] of health parameters in 235 calves $^{1}$ on arrival at a grain-fed veal facility in southwestern Ontario, Canada

\begin{tabular}{|c|c|c|c|c|}
\hline Health parameter & \multicolumn{4}{|c|}{ Score } \\
\hline Cough score & $\begin{array}{l}\text { No cough } \\
97 \%(226)\end{array}$ & $\begin{array}{l}\text { Single cough induced } \\
3 \%(7)\end{array}$ & $\begin{array}{l}\text { Occasional spontaneous or } \\
\text { repeated induced coughs } \\
0 \%(0)\end{array}$ & $\begin{array}{l}\text { Repeated spontaneous } \\
\text { coughs } \\
0 \%(0)\end{array}$ \\
\hline Flank score & $\begin{array}{l}\text { Normal } \\
50 \%(116)\end{array}$ & $\begin{array}{l}\text { Paralumbar fossa is } \\
\text { visibly depressed } \\
50 \%(116)\end{array}$ & & \\
\hline Other score & $\begin{array}{l}\text { No evidence of health } \\
\text { abnormality other than } \\
\text { those assessed } \\
99 \%(231)\end{array}$ & $\begin{array}{l}\text { Notable evidence of a } \\
\text { health abnormality not } \\
\text { otherwise assessed } \\
1 \%(2)\end{array}$ & & \\
\hline
\end{tabular}

${ }^{1}$ Health parameters were measured for 235 calves. Between 2 and 3 missing data points were found at random within the health parameter data set, leading to total calf numbers reported for each health parameter between 232 and 233 calves.

diagnostic accuracy study. A white blood cell manual differential test was performed for each calf by microscopy at the Animal Health Laboratory. The Animal Health Laboratory is accredited by the American Association of Veterinary Laboratory Diagnosticians, the Standards Council of Canada, and the Canadian Association for Laboratory Accreditation. Due to the large number of samples submitted, blood smears were made and fixed at the time of submission, but manual differential analysis was performed as time permitted. A modified Wright stain and $100 \times$ oil immersion were used to scan the monolayer of the stained blood smear, and cells were counted until a total of 100 cells was reached. The slides were scanned by technicians with identical training, standard operating procedures, and membership in the Veterinary Laboratory Association Quality Assurance Program (Atlantic Veterinary College, Charlottetown, PE, Canada). In addition, a subset of slides was reviewed daily by a board-certified clinical pathologist as part of routine internal quality assurance protocols. Individual cell concentrations were determined using the manual differential proportion and the total white blood cell concentration measured by the Advia 2120 hematology analyzer (Siemens Healthcare Ltd., Oakville, ON, Canada). The Advia 2120 machine at the Animal Health Laboratory was evaluated daily as part of an inter-laboratory quality assurance program to compare predetermined sample results between laboratories.

Health scores and measurements were not available to those performing either the QScout BLD or the manual differential tests. The results of the QScout BLD test were not available to those performing the manual differential tests.

The 10 -mL serum tubes were centrifuged at $2,188 \times$ $g$ for $15 \mathrm{~min}$ as soon as they arrived at the laboratory. Serum was stored in the refrigerator overnight and removed in the morning for serum total protein analysis. Samples were transferred to an optical refractometer (RHC-200ATC; SinoTech, Fujian, China) using a new disposable pipette for each sample. Values were visually determined, and results were recorded first on paper and then entered into a spreadsheet.

\section{Sample-Size Calculation}

Blood parameter data were assumed to have normal distribution and to be within $10 \%$ of the true upper and lower population interval. We used the method for sample-size determination described by Hahn and Meeker for tolerance intervals, assuming 95\% confidence in capturing $95 \%$ of the population (Hahn and 
Table 2. Descriptive statistics of blood parameters in 235 calves on arrival at a grain-fed veal facility in southwestern Ontario

\begin{tabular}{|c|c|c|c|c|c|c|c|c|}
\hline Variable & \multicolumn{4}{|c|}{ QScout value $^{1}$} & \multicolumn{4}{|c|}{ Manual value ${ }^{2}$} \\
\hline Neutrophils $\left(10^{9} / \mathrm{L}\right)$ & 5.70 & 3.07 & 0.21 & 19.24 & 5.15 & 2.94 & 0.48 & 22.79 \\
\hline Monocytes $\left(10^{9} / \mathrm{L}\right)$ & 0.03 & 0.06 & 0 & 0.59 & 0.90 & 0.57 & 0.04 & 2.99 \\
\hline Eosinophils $\left(10^{9} / \mathrm{L}\right)$ & 0.35 & 0.20 & 0 & 1.19 & 0.15 & 0.16 & 0 & 0.78 \\
\hline Basophils $\left(10^{9} / \mathrm{L}\right)$ & 0 & 0 & 0 & 0 & 0.12 & 0.05 & 0 & 0.26 \\
\hline
\end{tabular}

${ }^{1}$ Machine values as determined by QScout BLD (Advanced Animal Diagnostics, Morrisville, NC).

${ }^{2}$ Manual values as determined by the Animal Health Laboratory (Guelph, ON, Canada).

Meeker, 2011). This resulted in an estimated sample size of 173 calves.

\section{Analysis}

We performed statistical analyses using Stata 15 (StataCorp LP, College Station, TX). Data were imported to Stata 15 from a spreadsheet, and samples with missing data for either test were excluded from analysis. Descriptive statistics were generated for white blood cell differential count, health score, serum total protein, and calf rectal temperature. We used Lin's concordance correlation coefficient $(\boldsymbol{\rho})$ to measure the agreement between the QScout BLD and manual differential tests. We used the upper and lower 95\% reference intervals determined for neonatal Holstein calves (Panousis et al., 2018) to compare test agreement in the overall classification of the samples as above, below, or within normal limits for each white blood cell category.

\section{RESULTS}

\section{Animal Population}

The 240 calves arriving at the grain-fed veal facility were Holstein male calves of unknown age (estimated to be 3 to $7 \mathrm{~d}$ of age at arrival) and history. All calves were eligible for enrollment at arrival. Of the 240 calves that arrived, 5 were excluded from the data set. Calves were removed from the data set for having a missing blood test value $(\mathrm{n}=1)$, an invalid calf identification number $(\mathrm{n}=3)$, or a QScout differential test that did not return results $(\mathrm{n}=1)$. A total of 235 calves were included in the final data set.

Mean serum total protein for the calves on the day of arrival was $5.5 \mathrm{~g} / \mathrm{dL}$ (SD $0.72 \mathrm{~g} / \mathrm{dL}$ ); $26.3 \%$ of calves were below a cutoff of $5.1 \mathrm{mg} / \mathrm{dL}$, indicating failed passive transfer of immunity (Renaud et al., 2018b). A total of 65 calves $(27.1 \%)$ had a rectal temperature $>39.5^{\circ} \mathrm{C}$, indicating a fever. The mean rectal tempera- ture was $39.4^{\circ} \mathrm{C}\left(\mathrm{SD} 0.42^{\circ} \mathrm{C}\right)$. Table 1 describes the proportion of calves by score for the health parameters. Approximately a quarter of the calves arrived at the facility with mild dehydration, and half the calves had a sunken paralumbar fossa, which we believe to be indicative of a prolonged period since their last feeding. Approximately $29 \%$ of the calves arrived with an abnormal navel score, but less than $5 \%$ of calves arrived with a cough or other detectable health abnormality.

\section{Test Results}

Descriptive statistics for both tests are presented in Table 2. Concordance plots between the tests for neutrophil, lymphocyte, monocyte, and eosinophil counts and N:L are shown in Figure 1.

We demonstrated very good agreement between the QScout BLD and manual differential test for neutrophil count $(\rho=0.83,95 \%$ CI $0.79-0.87, P<0.0001]$; this agreement can be visualized as a tight cluster of data points around the equality line in Figure 1A. We demonstrated fair agreement between tests for lymphocyte count $(\rho=0.32,95 \%$ CI $0.25-0.40, P<0.0001)$; the data points were much more dispersed around the line of equality, indicating a lack of precision (Figure 1B). We observed only slight agreement for monocytes $(\rho=$ $0.14,95 \%$ CI $0.01-0.18, P<0.0001]$ and eosinophils $(\rho$ $=0.20,95 \%$ CI $0.02-0.373, P<0.026)$; in general, the data were fairly well clustered around the line of concordance, which may indicate good test precision for measuring monocytes and eosinophils (Figure 1C and Figure 1D). We demonstrated fair agreement between tests for N:L ratio $(\rho=0.36,95 \%$ CI $0.3-0.42, P<$ 0.0001]; the data points were well clustered around the line of concordance, with the exception of N:L ratios larger than 4, which appeared more dispersed (Figure $1 \mathrm{E})$.

We plotted the QScout BLD versus manual differential test results with the upper and lower reference interval lines on both axes to evaluate test classification 
A

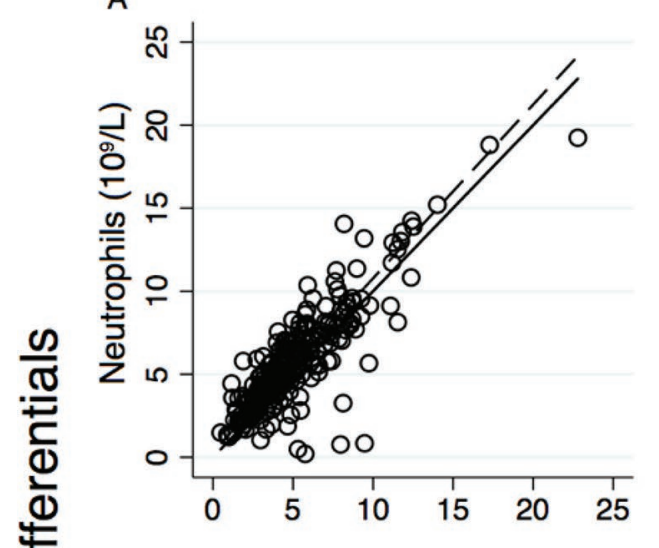

B

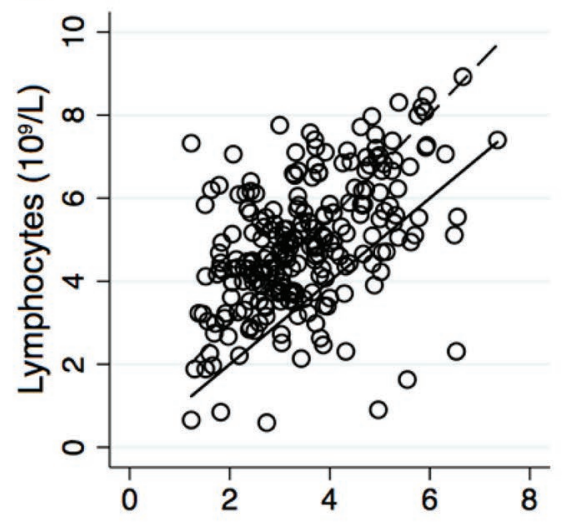

C

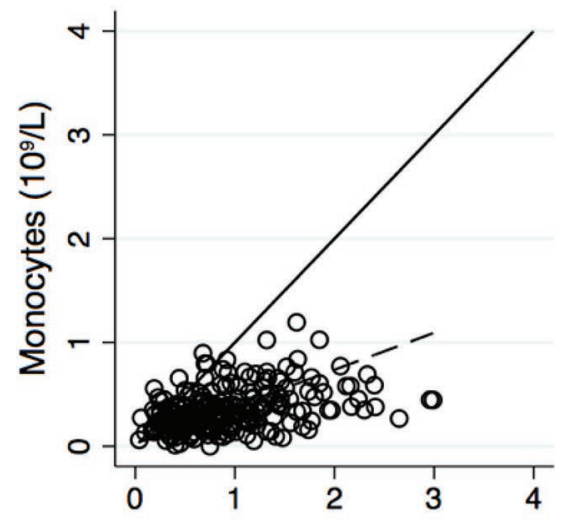

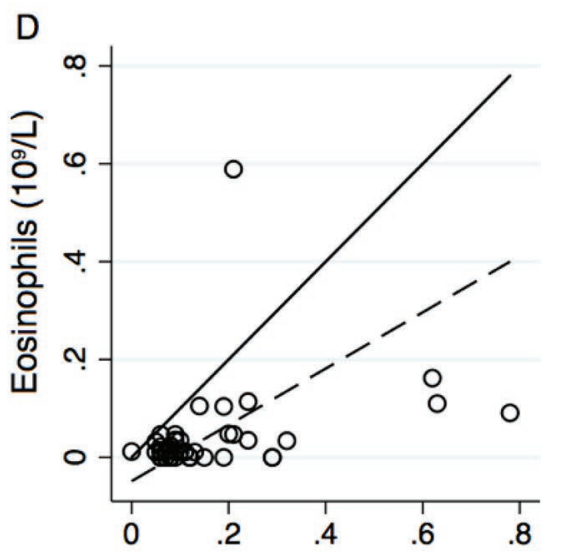

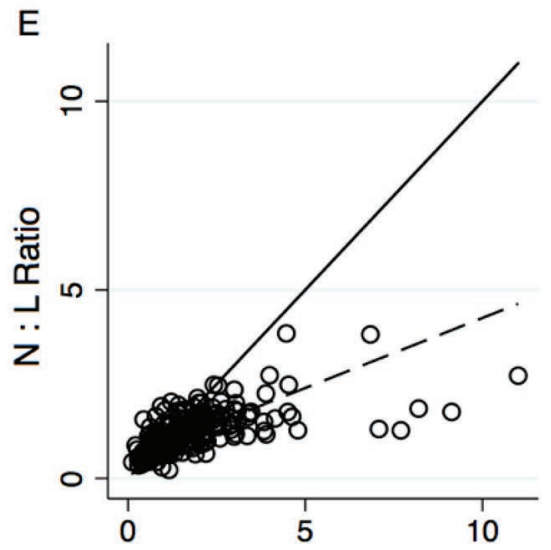

Manual Differentials

Figure 1. Agreement between manual and machine (QScout BLD; Advanced Animal Diagnostics, Morrisville, NC) white blood cell differentials collected from 235 calves on arrival at a grain-fed veal facility in southwestern Ontario, Canada. Agreement is presented for (A) neutrophils, (B) lymphocytes, (C) monocytes, (D) eosinophils, and (E) neutrophil:lymphocyte ratio (N:L). The solid black line represents the line of perfect concordance; the dashed line represents the observed concordance of the data.

of blood parameters as high, low, or normal (Figure 2). For neutrophils, most test results fell within the normal reference intervals, with only $4.2 \%$ disagreement between tests (Figure 2A). For lymphocytes, the majority of test results were classified within normal limits by both tests, with $5.8 \%$ disagreement between tests (Figure 2B). The QScout BLD test appeared to trend toward lower monocyte counts compared to the manual differential test, with $23.3 \%$ disagreement between tests (Figure 2C). The QScout BLD test also appeared to trend toward lower eosinophil counts compared to the manual differential test, with $70.8 \%$ disagreement between tests (Figure 2D). For N:L ratio, most test results fell within the normal reference intervals, with only $3.0 \%$ disagreement between tests (Figure 2E). Considering the manual differential blood values alone, $2.1 \%$ of calves tested had total leukocyte counts outside of the established reference intervals for neonatal
Holstein calves, and $2.6 \%$ had N:L ratios outside the reference interval (Figure 2E).

\section{DISCUSSION}

Although manual leukocyte differential counts remain the gold standard, automated cell counts are rapid and reproducible, and they have a strong correlation with most leukocyte cell lines (Kim et al., 2014). This study demonstrates that in neonatal Holstein calves, the QScout BLD test had very good agreement with manual leukocyte differential results for neutrophil counts, fair agreement for lymphocyte counts, but only slight agreement for eosinophils and monocytes, consistent with findings in Holstein cows (Kull et al., 2018). The classification of test results as above, below, or within reference intervals for neutrophils and lymphocytes was in very close agreement between tests. However, 
the QScout BLD test appeared to trend toward lower counts for both monocytes and (to a greater extent) eosinophils, resulting in classification disagreements in over a fifth of the samples for monocytes and for the majority of samples for eosinophils. The QScout BLD software algorithm used to differentiate between leukocyte cell lines continues to be refined and improved, with new versions in development.

A possible limitation of this study was the time between sample acquisition and the manual differential test. The manual differential test was not processed until approximately $12 \mathrm{~h}$ after sample collection, because of distance from the laboratory and the submission of the samples occurring outside laboratory hours of operation. A decrease in monocyte concentration and an increase in lymphocyte and eosinophil concentration has been observed in bovine blood samples stored in EDTA anticoagulant at $4^{\circ} \mathrm{C}$ for up to $72 \mathrm{~h}$ after collection, but these changes were relatively small and unlikely to alter interpretation of results (Warren et al., 2013). These small changes are associated with cellular degradation over time that can result in misclassification of monocytes and large unstained cells as lymphocytes, as well as a mild increase in eosinophil and overall leukocyte counts by the Advia hematology analyzer (Warren et al., 2013). In the literature, it is recommended that bovine leukocytes be examined in samples stored in EDTA at $4^{\circ} \mathrm{C}$ within $24 \mathrm{~h}$ after collection, because the effect on results is expected to be minimal (Jones and Allison, 2007; Warren et al., 2013; Roland et al., 2014).

Monocyte and eosinophil numbers showed only slight agreement between the QScout BLD and manual differential tests. However, the utility of these values in neonatal calves might be limited. Eosinophil numbers in calves are low at birth and only begin to increase after 3 wk of age (Jones and Allison, 2007; Tornquist and Rigas, 2011; Roland et al., 2014), making this an
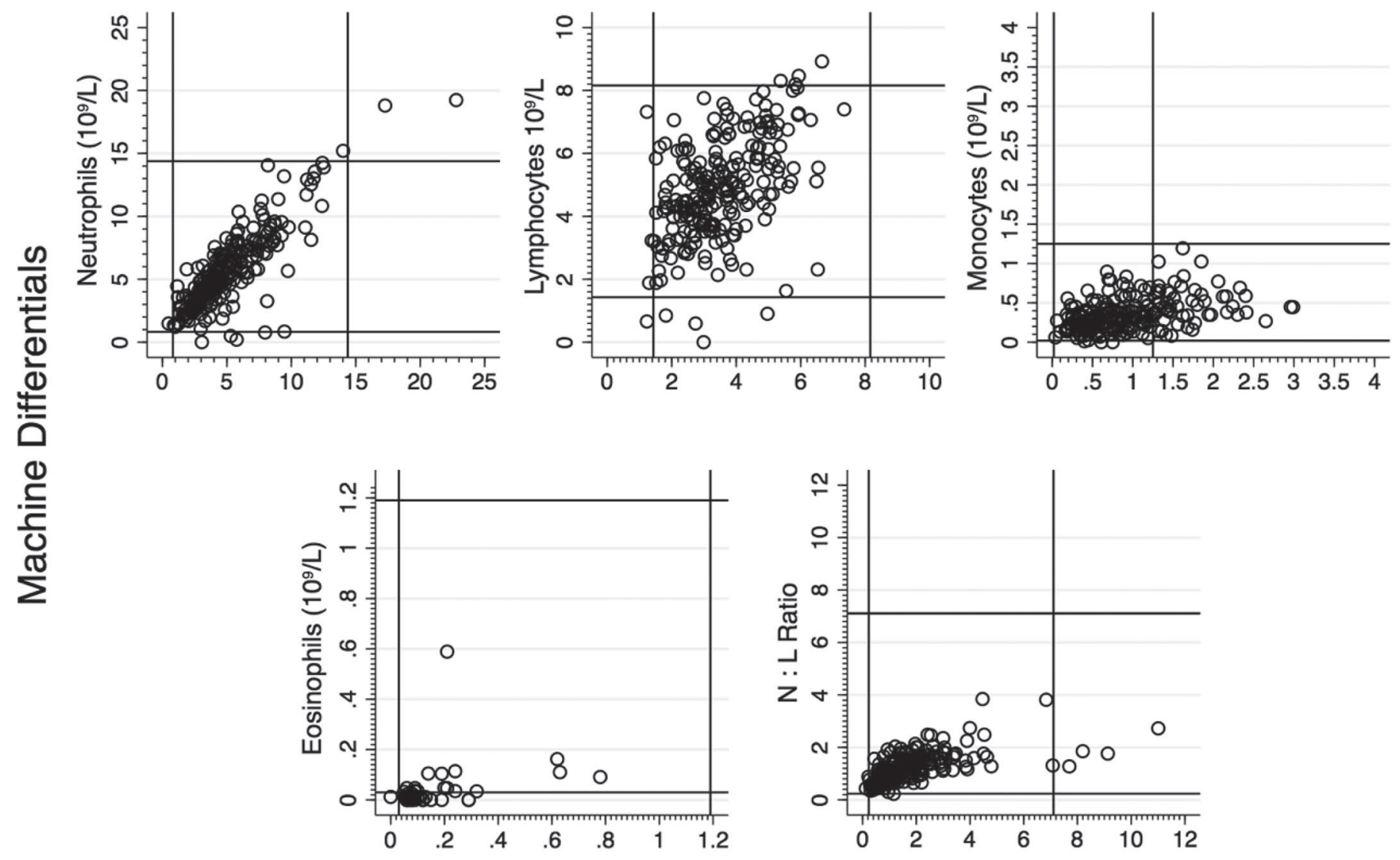

\section{Manual Differentials}

Figure 2. Manual versus machine (QScout BLD; Advanced Animal Diagnostics, Morrisville, NC) white blood cell differentials collected from 235 calves on arrival at a grain-fed veal facility in southwestern Ontario, Canada. Results are presented for (A) neutrophils, (B) lymphocytes, (C) monocytes, (D) eosinophils, and (E) neutrophil:lymphocyte ratio (N:L). The black lines represent the hematological reference intervals for neonatal Holstein calves (Panousis et al., 2018). 
unreliable health indicator in neonatal Holstein calves (estimated to be between 3 and $7 \mathrm{~d}$ of age). Additionally, monocyte responses are variable in cattle (Jones and Allison, 2007; Tornquist and Rigas, 2011; Roland et al., 2014) and may not be a sensitive indicator of specific pathologic processes (Jones and Allison, 2007; Tornquist and Rigas, 2011).

Prolonged stress, such as stress during transportation, can result in impaired immune function due to glucocorticoid exposure, resulting in significant neutrophilia, lymphopenia, and overall leukopenia, with an increase in the N:L ratio (Jones and Allison, 2007; Tornquist and Rigas, 2011; Marcato et al., 2018). Changes noted in $\mathrm{N}: \mathrm{L}$ ratios along with overall leukocyte counts may be sufficient to differentiate between stress, inflammatory, and excitement leukograms in combination with clinical exam findings. The N:L ratio in calves is $>1$ in the first week of life due to higher neutrophil counts, and approaches that of adults (0.5) by approximately 1 wk of age (Tornquist and Rigas, 2011).

Temporary fear or excitement can cause a mild and transient increase in both neutrophils and lymphocytes, as well as overall leukocytosis (Jones and Allison, 2007; Tornquist and Rigas, 2011), which may not have a significant measurable effect on the N:L ratio. Acute inflammation can result in initial neutropenia due to neutrophil recruitment into tissues and decreased N:L ratio, but chronic inflammation or resolution of inflammation can result in a return to normal neutrophil numbers and N:L ratio after 4 to $5 \mathrm{~d}$ (Jones and Allison, 2007; Tornquist and Rigas, 2011), highlighting the importance of clinical exam findings and overall leukocyte counts in addition to N:L ratio. In this study, less than $3 \%$ of calves had total leukocyte counts or $\mathrm{N}: \mathrm{L}$ ratios outside the reported reference interval, even though over $72 \%$ of the population arrived with at least 1 health parameter score above normal. As a result of the small number of abnormal leukograms measured in this study, it was not possible to evaluate the ability of the QScout BLD to correctly identify them.

White blood cell differentials are seldom used alone when making a diagnosis (Roland et al., 2014). Further study of the leukocyte profiles of calves arriving at veal facilities and how they relate to concurrent or subsequent morbidity and mortality in the growing period is required. Recent advances in identifying high-risk calves at arrival through clinical examination and biomarkers (Renaud et al., 2017a,b; Marcato et al., 2018) are helping to build a risk profile for calves of unknown age and history. Should the leukocyte profiles of these calves prove to be an accurate indicator of future risk, then a rapid, calf-side automated leukocyte differential test would present a promising addition to augmenting or refining risk identification for implementing selective antimicrobial therapy protocols at arrival.

\section{CONCLUSIONS}

The automated cell counter, QScout BLD, performed well in estimating neutrophil differential counts, fairly for lymphocyte counts and N:L ratio, and less well for monocyte and eosinophil counts. Total leukocyte counts and N:L ratios, in combination with clinical exam findings, have the potential to provide sufficient information to differentiate between stress, inflammation, and temporary fear or excitement in leukocyte differential results. Further study of the leukocyte profile of neonatal calves arriving at veal and dairy beef facilities is necessary to determine its utility in assessing the risk of morbidity and mortality.

\section{ACKNOWLEDGMENTS}

The authors thank the research assistants involved in data collection and the farm staff for their cooperation. The authors also thank the Ontario Ministry of Agriculture, Food and Rural Affairs (OMAFRA, Guelph, ON, Canada), the Veal Farmers of Ontario (Guelph, ON, Canada), the Ontario Veterinary College (University of Guelph, ON, Canada), Mapleview Agri Ltd. (Palmerston, ON, Canada), and Advanced Animal Diagnostics (Morrisville, NC) for their support.

\section{REFERENCES}

Bähler, C., A. Steiner, A. Luginbühl, A. Ewy, H. Posthaus, D. Strabel, T. Kaufmann, and G. Regula. 2012. Risk factors for death and unwanted early slaughter in Swiss veal calves kept at a specific animal welfare standard. Res. Vet. Sci. 92:162-168. https://doi .org/10.1016/j.rvsc.2010.10.009.

Bos, M. E., F. J. Taverne, I. M. van Geijlswijk, J. W. Mouton, D. J. Mevius, D. J. Heederik, and N. S. Da. 2013. Consumption of antimicrobials in pigs, veal calves, and broilers in the Netherlands: Quantitative results of nationwide collection of data in 2011. PLoS One 8:e77525. https://doi.org/10.1371/journal.pone.0077525.

Catry, B., J. Dewulf, D. Maes, B. Pardon, B. Callens, M. Vanrobaeys, G. Opsomer, A. de Kruif, and F. Haesebrouck. 2016. Effect of antimicrobial consumption and production type on antibacterial resistance in the bovine respiratory and digestive tract. PLoS One 11:e0146488. https://doi.org/10.1371/journal.pone.0146488.

Hahn, G. J., and W. Q. Meeker. 2011. Statistical Intervals: A Guide for Practitioners. John Wiley \& Sons, Inc., New York, NY. https: //doi.org/10.1002/9780470316771.ch6.

Jones, M. L., and R. W. Allison. 2007. Evaluation of the ruminant complete blood cell count. Vet. Clin. North Am. Food Anim. Pract. 23:377-402. https://doi.org/10.1016/j.cvfa.2007.07.002.

Kim, A. H., W. Lee, M. Kim, Y. Kim, and K. Han. 2014. White blood cell differential counts in severely leukopenic samples: A comparative analysis of different solutions available in modern laboratory hematology. Blood Res. 49:120-126. https://doi.org/10.5045/br .2014.49.2.120.

Korevaar, D. A., J. F. Cohen, J. B. Reitsma, D. E. Bruns, C. A. Gatsonis, P. P. Glasziou, L. Irwig, D. Moher, H. C. W. de Vet, 
D. G. Altman, L. Hooft, and P. M. M. Bossuyt. 2016. Updating standards for reporting diagnostic accuracy: The development of STARD 2015. Res. Integr. Peer Rev. 1:7. https://doi.org/10.1186/ s41073-016-0014-7.

Kull, J. A., P. D. Krawczel, and G. M. Pighetti. 2018. Short communication: Evaluation of an automated method for assessing white blood cell concentrations in Holstein dairy cows. Vet. Immunol. Immunopathol. 197:21-23. https://doi.org/10.1016/j.vetimm.2018 .01 .002

Marcato, F., H. van den Brand, B. Kemp, and K. van Reenen. 2018. Evaluating potential biomarkers of health and performance in veal calves. Front. Vet. Sci. 5:133. https://doi.org/10.3389/fvets.2018 .00133 .

McGuirk, S. M., and S. F. Peek. 2014. Timely diagnosis of dairy calf respiratory disease using a standardized scoring system. Anim. Health Res. Rev. 15:145-147. https://doi.org/10.1017/ S1466252314000267.

Mohri, M., K. Sharifi, and S. Eidi. 2007. Hematology and serum biochemistry of Holstein dairy calves: Age related changes and comparison with blood composition in adults. Res. Vet. Sci. 83:30-39. https://doi.org/10.1016/j.rvsc.2006.10.017.

Panousis, N., N. Siachos, G. Kitkas, E. Kalaitzakis, M. Kritsepi-Konstantinou, and G. E. Valergakis. 2018. Hematology reference intervals for neonatal Holstein calves. Res. Vet. Sci. 118:1-10. https:// doi.org/10.1016/j.rvsc.2018.01.002.
Renaud, D. L., T. F. Duffield, S. J. LeBlanc, S. Ferguson, D. B. Haley, and D. F. Kelton. 2018a. Risk factors associated with mortality at a milk-fed veal calf facility: A prospective cohort study. J. Dairy Sci. 101:2659-2668. https://doi.org/10.3168/jds.2017-13581.

Renaud, D. L., T. F. Duffield, S. J. LeBlanc, D. B. Haley, and D. F. Kelton. 2018b. Clinical and metabolic indicators associated with early mortality at a milk-fed veal facility: A prospective case-control study. J. Dairy Sci. 101:2669-2678. https://doi.org/10.3168/ jds.2017-14042.

Roland, L., M. Drillich, and M. Iwersen. 2014. Hematology as a diagnostic tool in bovine medicine. J. Vet. Diagn. Invest. 26:592-598. https://doi.org/10.1177/1040638714546490.

Tornquist, S. J., and J. Rigas. 2011. Schalm's veterinary hematology. Vet. Clin. Pathol. 40:307-313. https://doi.org/10.1111/j.1939 $-165 x .2011 .00324 . x$.

Warren, A. L., T. Stokol, K. G. Hecker, and D. V. Nydam. 2013. Storage-associated changes in the bovine hemogram with the ADVIA 120 hematology analyzer. Comp. Clin. Pathol. 22:1235-1240. https://doi.org/10.1007/s00580-012-1556-9.

Winder, C. B., D. F. Kelton, and T. F. Duffield. 2016. Mortality risk factors for calves entering a multi-location white veal farm in Ontario, Canada. J. Dairy Sci. 99:10174-10181. https://doi.org/10 $.3168 /$ jds.2016-11345. 